\title{
Risk factors for post-endoscopic retrograde cholangiopancreatography pancreatitis: Evidence from 810 cases
}

\author{
(1) Bahtiyar Muhammedoğlu \\ Department of Gastroenterological Surgery, Necip Fazıl City Hospital, Kahramanmaraş, Turkey
}

\begin{abstract}
Introduction: The risk and causes of post ERCP pancreatitis and associated risk factors are summarized, and potential prophylactic measures with strong evidence for effective prevention of post ERCP pancreatitis are discussed.

Materials and Methods: Prospectively collected patient data were reviewed retrospectively for a total of 810 ERCPs undertaken in our hospital between June 2015 and September 2018. All ERCP procedures were performed by a single surgeon. Risk factors for post-ERCP pancreatitis were investigated, which included endoscopist experience, ERCP team and ERCP room equipment. We focused on factors related to endoscopist experience, ERCP team and ERCP room equipment.

Results: A total of 810 patients, including 439 females and 371 males, were enrolled in this study. Amylase levels and pancreatitis were evaluated 24 hours after ERCP. Post ERCP pancreatitis developed in a total of 46 (5.5\%) patients out of 810 patients undergoing ERCP. Also, the incidence of post ERCP pancreatitis was 2 -fold higher in the first 400 patients versus in the last 400 patients. This higher post ERCP pancreatitis incidence found among the first 400 patients of our series can be explained by the relative lack of experience and expertise of the endoscopist and the ERCP team.
\end{abstract}

Conclusion: The risk of post-ERCP pancreatitis is multifactorial and the effects of some of the risk factors may be minimized or completely eliminated. The findings suggest that endoscopist and his/her experience have a major role in avoiding or minimizing the negative effects of these factors.

Keywords: Endoscopy; hyperamylasemia; patients.

\section{Introduction}

The overall incidence of post-endoscopic retrograde cholangiopancreatography (ERCP) pancreatitis has been reported to be approximately $3 \%$ to $15 \%$ in recent studies. Known risk factors include patient- and ERCP-related risks. Prevention or minimization of risk factors during ERCP is of great importance for post-ERCP pancreatitis (PEP) prophylaxis. ERCP is increasingly used in the therapeutic management of various biliary and pancreatic diseases. ${ }^{[1]}$ 
The effects of mechanical trauma resulting from repeated attempts at biliary cannulation and injection of contrast agent during ERCP have been commonly reported. There are a number patient-related (e.g. Sphincter of Oddi dysfunction, female gender, younger age, normal serum bilirubin) and procedure-related (e.g. difficult cannulation, papillary balloon dilation, pancreatic sphincterotomy, pancreatic duct injection) risk factors for PEP. Unfortunately, PEP carries a potential risk for morbidity as well as occasional mortality. ${ }^{[2]}$ In some cases, pancreatic necrosis results in prolonged treatment, disability, and even death. PEP is a predictable pathology, and if diagnosed and appropriately treated in an early stage, many patients rapidly recover. ${ }^{[3]}$ The aim of the present study was to review risk factors for post-ERCP pancreatitis retrospectively. At the same time, our aim of this study was to compare the groups according to serum amylase value and to evaluate the liver function test.

\section{Materials and Methods}

Study population prospectively collected patient data were reviewed retrospectively for a total of 810 ERCPs undertaken in our hospital between June 2015 and September 2018. All ERCP procedures were performed by a single surgeon. As the study was retrospective, the Institutional Review Board (IRB) approval did not need. Risk factors for post-ERCP pancreatitis were investigated which included endoscopist experience, ERCP team and ERCP room equipment.

Groups were compared according to serum amylase values $<300$ and $>300$ and liver function tests were evaluated. In the first 400 and last 410 patients, PEP was evaluated. Routine preoperative strategies were consistent with standard pre-ERCP procedures. Prior to ERCP, patients received a NSAID (Diclofenac, $75 \mathrm{mg}$ intramuscularly) and prophylactic antibiotic (cefoperazone, $2 \mathrm{~g} / \mathrm{d}$ ) therapy as well as standard intravenous hydration with lactated Ringer's solution $(1.5 \mathrm{~mL} / \mathrm{kg} / \mathrm{h}$ during and for 8 hours after ERCP) at our clinic. Vital signs were obtained $24 \mathrm{~h}$ after completion of ERCP. Demographic characteristics, post-ERCP pancreatitis, the disease severity and mortality were recorded for all patients. In the current study, we focused on the factors related to the endoscopist, ERCP team and ERCP room equipment. In our study After the ERCP procedure, pancreatitis was defined as mild, moderate and severe, and the following identification criteria were identified. Mild PEP: when the patient developed abdominal pain, amylase or lipase elevation to $\geq 3$ times the upper limit of normal, without evidence of organ dysfunction or complications more than 24 hours after ERCP. Moderate PEP: when the patient developed with transient ( $<48$ hours) organ failure or local or systemic complications without persistent organ failure. Severe PEP: was defined as PEP with persistent single or multi-organ failure ( $>48$ hours) or present or persistent systemic inflammatory response syndrome (SIRS).

\section{Factors Related to ERCP Procedure}

Our clinic has an ERCP unit which greatly contributes to the management of hepatobiliary disorders. In addition to routine treatment practices, advanced ERCP procedures are actively performed in our clinic. Before the surgical procedure, all patients undergo a thorough assessment of possible risk factors for PEP. Anatomical features of the papilla differ between patients and selective cannulation of the common bile duct may be difficult even for experienced endoscopists during ERCP. Every unsuccessful attempt at cannulation is associated with an increased risk of post-ERCP pancreatitis. Injection of contrast medium into the pancreatic duct is an independent predictor of PEP. Therefore, the wire-guided biliary cannulation technique is used to avoid unnecessary pancreatography. Meta-analyses have shown that the wire-guided cannulation technique was associated with greater primary cannulation success and the incidence of PEP was significantly lower with the wire-guided technique as compared with the standard contrast-assisted cannulation technique. ${ }^{[4,5]}$ Guided by X-ray imaging, the guidewire should be pushed gently and fit into the imaginary lines of CBD and Wirsung duct. Endoscopist may develop a guidewire cannulation technique for the biliary duct by reviewing MRCP images prior to the procedure. The guidewire-assisted technique reduces the need for the use of contrast medium which is itself associated with an increased risk for post-ERCP pancreatitis. It should be remembered that team work principle is among the most important factors. An experienced radiology technician and a high-quality $\mathrm{C}$ arm X-ray imaging system are integral to obtain optimal images. Quality X-ray imaging, appropriate patient position (prone or left lateral decubitus position) and guidewire-assisted CBD cannulation are associated with increased ERCP success and reduce the risk of PEP by avoiding inadvertent cannnulations of the Wirsung duct. Additionally, the need for using contrast agents is reduced with increased experience and expertise of the surgeon. 


\section{Risk Factors of Post-ERCP Pancreatitis}

Revised Atlanta classification was used for the diagnosis and grading of PEP. PEP was diagnosed following the Revised Atlanta Classification's criteria. Mild PEP was considered when the patient developed abdominal pain, amylase or lipase elevation to $\geq 3$ times the upper limit of normal, without evidence of organ dysfunction or complications more than 24 hours after ERCP. Moderate PEP was defined as PEP with transient ( $<48$ hours) organ failure or local or systemic complications without persistent organ failure. Severe PEP was defined as PEP with persistent single or multi-organ failure ( $>48$ hours) or present or persistent systemic inflammatory response syndrome (SIRS). ${ }^{[6]}$ Widely recognized risk factors for post-ERCP pancreatitis can be broadly divided into two categories: patient- and procedure-related. As can be seen from Figure 1, all of these factors are important for the development of PEP and may cause PEP either individually or in combination. Even when ERCP procedure fails, timely completion of the ERCP is essential to avoid complications.

\section{Patient-Related Risk Factors}

High-risk patients may develop PEP independently of the type of endoscopic procedure employed. Also, the coexistence of multiple risk factors in a single patient is associated with an increased risk of PEP. Patient-related factors include younger age ( $<40$ years), suspected sphincter of Oddi dysfunction (SOD), history of previous post-ERCP pancreatitis, peripapillary duodenal diverticulum and normal serum bilirubin. Pancreatic stent placement should be considered particularly in the following conditions: pre-cut sphincterotomy; sphincter of Oddi dysfunction, transpancreatic septotomy, suspected SOD, pancreatic brush cytology, baloon dilation, and subsequent to a difficult cannulation or repeated contrast agent injections into the pancreatic duct. It is preferable to use a prophylactic pancreatic stent with a small diameter (4-5 French). Procedure-related factors include papillary trauma from repeated attempts of cannulation, injection of contrast medium into the pancreatic duct and pancreatic sphincterotomy (septotomy). These complications may potentially be avoided when the procedure is performed by an experienced endoscopist. However, studies exist in literature which reported no correlation of endoscopist experience with the development of PEP.

\section{Endoscopist Experience}

A trainee must continuously learn and practice this technique to become an experienced ERCP endoscopist.

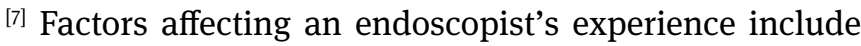
skill, manual dexterity, individual and institutional conditions, ERCP/EST experience and frequency. ERCP frequency and experience are equally important as individual and institutional conditions. ${ }^{[8]}$ In order to achieve competency, fellows have to complete ERCP training and at least 100 procedures. ${ }^{[9]}$ In a large-scale study that compared the experience of endoscopy centers and providers, no difference was found between high-volume and lowvolume centers in the incidence of post-ERCP pancreatitis after 3635 ERCP procedures. Also, that study did not find a statistically significant difference in the rates of post-ERCP pancreatitis between expert and non-expert endoscopists. ${ }^{[10]}$ Some studies reported that a pre-ERCP multidisciplinary team meeting reduced the frequency of post-ERCP complications and concluded that such meetings should be recommended in the clinical practice. ${ }^{[1]}$ Anesthesia services have been increasingly used in recent years to achieve deep sedation. All of our patients were evaluated by anesthesiologists prior to ERCP using the American Society of Anesthesiologists (ASA) physical status classification system. Biliary duct cannulation is facilitated in a patient by adequate sedation. However, ERCP may become more difficult with inadequate sedation because of patient agitation, leading to unsuccessful premature termination of the procedure and potential adverse events.

\section{ERCP Room and Team}

ERCP room should comply with generally accepted standards. Radiology technician should be experienced in obtaining images with C-arm fluoroscopic X-ray system. While there are several C-arm devices available for use, the presence of a high-quality, state-of-the art C-arm fluoroscopic X-ray system in the ERCP suite further facilitates the procedure. An experienced radiology technician and necessary equipment should be present to obtain these images. Availability of internet connection and a recording and HD imaging system in the ERCP room and proper distance of the endoscopist to the image screen allow fast and accurate interpretation of cholangiographs. ERCP nurse also has a significant role as a key member of the ERCP team. Close coordination between the ERCP nurse and the endoscopist helps avoid ERCP complications and increases the success of ERCP. 


\section{Internet Applications}

Clinical manifestations, MRCP images and laboratory results may not be sufficient to determine the indication for an ERCP in every patient. Even an experienced endoscopist may have difficulties in the management of some patients. MRCP is important for the diagnosis of biliary disorders and requires high-quality scanning and good image interpretation. Endoscopists may discuss a case with fellow endoscopists who are interested in ERCP by sharing patient data and images through internet applications and mutually contribute to patient management. When there was the slightest doubt regarding the indication of our patients or consensus was sought for patient management, we facilitated the management of our patients by discussing cases with a friendly team of doctors via internet applications. In some cases, ERCP procedures were performed through real-time image sharing. The infrastructure of our ERCP unit allows such interaction. Discussion of patient cases with a friendly team of doctors through data exchange helps all doctors involved in the discussion gain further experience.

\section{Alternative Techniques}

Cannulation may be challenging in 10 to $20 \%$ of cases even in the experienced hands due to patient- or procedure-related factors. ${ }^{[12]}$ Precut sphincterotomy significantly increases the rate of biliary cannulation, up to $98 \% .{ }^{[13]}$ Very rarely, we selectively cannulated the common bile duct with transpancreatic septotomy. We believe that, in addition to known PEP risk factors, ERCP room, ERCP team and endoscopist experience also play an important role in the development of PEP. Recent evidence suggests that precut sphincterotomy alone may not be a risk factor for pancreatitis; rather repeated attempts $(\geq 10)$ at biliary cannulation prior to precut sphincterotomy may be the actual cause of post-ERCP pancreatitis. ${ }^{[13]}$ Experienced endoscopists avoid persistent attempts in the case of difficult CBD cannulation and employ alternative cannulation techniques; this approach reduces the duration of ERCP. ${ }^{[14]}$ In the present study, we performed fistulotomy or pre-cut sphincterotomy due to difficult biliary cannulation after failure of 6 cannulation attempts or total cannulation time greater than 10 minutes. For patients with failed selective CBD cannulation despite use of alternative techniques, we terminated and rescheduled the ERCP procedure.

\section{Outcome Assessment}

The primary outcome measures were the comparison of PEP rates between the two groups. Secondary outcome measures were to evaluate prevention or minimization of risk factors during ERCP. Post ERCP pancreatitis was evaluated according to clinical signs and amylase values. At the same time, our aim of this study was to compare the groups according to serum amylase value and to evaluate the liver function test.

\section{Statistical Analysis}

The normality of distribution of continuous variables was tested by Shaphiro Wilk test. Mann-Whitney U test was used to compare 2 independent group for non-normal data and Kruskal Wallis and Dunn multiple comparison test were applied for three group comparisons. Chi-square test applied to investigate relationship between 2 categorical variables. Statistical analysis was performed with SPSS for Windows version 24.0 and a $P$ value $<0.05$ was accepted as statistically significant.

\section{Results}

A total of 810 patients including 439 females and 371 males were enrolled in this study (Table 1). Of them, 798 (98.5\%) underwent therapeutic ERCP and 16 (2\%) underwent diagnostic ERCP. The mean age of 810 patients was $61.66 \pm 18.92$ years and $48 \%$ of them were over 65 years of age. The most common ERCP indication was choledocholithiasis (93.9\%). Deep CBD cannulation was successful in 778 patients (96\%). For the remaining patients, precut sphincterotomy was performed due to failure of cannulation and ERCP was delayed for 72 hours. Biliary cannulation was performed during repeated ERCP. Despite second ERCP, biliary cannulation failed in 4 patients $(0.5 \%)$. At 24 hours after ERCP, amylase level was 3 times greater than the upper limit of normal in 285 patients (35.1\%), which was considered as postERCP hyperamylasemia. PEP developed in a total of 46 (5.5\%) out of 810 patients undergoing ERCP. Of these 46 patients, clinical severity of PEP was graded as mild in $28(60.8 \%)$ patients, moderate in $17(36.9 \%)$ patients and severe in 1 patient (2.17\%). Elevations of liver function tests including gamma-glutamyl transferase (GGT), direct bilirubin, alanine aminotransferase (ALT) and aspartate aminotransferase (AST) were statistically significant in the group with serum amylase values greater than $>300$ $\mathrm{IU} / \mathrm{L}(\mathrm{p}=0.001)$ (Table 2). Amylase value was significantly 
Table 1. Comparison of groups based on serum amylase value (cut-off of $300 \mathrm{IU} / \mathrm{L}$ )

\begin{tabular}{|c|c|c|c|c|c|}
\hline \multirow[t]{2}{*}{ Variables } & \multicolumn{2}{|c|}{$\begin{array}{c}\text { Amylase }<300 \text { IU/L } \\
(n=525)\end{array}$} & \multicolumn{2}{|c|}{$\begin{array}{c}\text { Amylase } \geq 300 \mathrm{IU} / \mathrm{L} \\
(\mathrm{n}=285)\end{array}$} & \multirow[t]{2}{*}{$\mathbf{p}$} \\
\hline & $\mathbf{n}$ & $\%$ & $\mathbf{n}$ & $\%$ & \\
\hline \multicolumn{6}{|l|}{ Gender } \\
\hline Male & 240 & 45.7 & 131 & 46.0 & 0.945 \\
\hline Female & 285 & 54.3 & 154 & 54.0 & \\
\hline \multicolumn{6}{|c|}{ Cholangiocellular carcinoma } \\
\hline Yes & 7 & 1.3 & 10 & 3.5 & $0.039 *$ \\
\hline No & 518 & 98.7 & 275 & 96.5 & \\
\hline \multicolumn{6}{|c|}{ Duodenal diverticula } \\
\hline Yes & 58 & 11.0 & 33 & 11.6 & 0.819 \\
\hline No & 467 & 89.0 & 252 & 88.4 & \\
\hline \multicolumn{6}{|c|}{ Suspected malignancy } \\
\hline Yes & 16 & 3.0 & 16 & 5.6 & 0.073 \\
\hline No & 509 & 7.0 & 269 & 94.4 & \\
\hline \multicolumn{6}{|l|}{ Bile leak } \\
\hline Yes & 3 & 0.6 & 6 & 2.1 & $0.047 *$ \\
\hline No & 522 & 99.4 & 279 & 97.9 & \\
\hline \multicolumn{6}{|c|}{ Second ERCP } \\
\hline Yes & 123 & 23.4 & 52 & 18.2 & 0.087 \\
\hline No & 402 & 76.6 & 233 & 81.8 & \\
\hline \multicolumn{6}{|c|}{ Placement of CBD stent } \\
\hline Yes & 97 & 18.5 & 66 & 23.2 & 0.112 \\
\hline No & 428 & 81.5 & 219 & 76.8 & \\
\hline \multicolumn{6}{|c|}{ Cholecystectomy } \\
\hline Yes & 51 & 9.7 & 36 & 12.6 & 0.200 \\
\hline No & 474 & 90.3 & 249 & 87.4 & \\
\hline \multicolumn{6}{|c|}{ Endoscopic sphincterotomy } \\
\hline Yes & 496 & 94.5 & 268 & 94.0 & 0.796 \\
\hline No & 29 & 5.5 & 17 & 6.0 & \\
\hline \multicolumn{6}{|c|}{ Endoscopic sclerotherapy } \\
\hline Yes & 14 & 2.7 & 5 & 1.8 & 0.413 \\
\hline No & 511 & 97.3 & 280 & 98.2 & \\
\hline
\end{tabular}

*Significant at 0.05 level. Chi-square test; ERCP. Endoscopic retrograde cholangiopancreatography; CBD: Common bile duct.

higher in the first 400 patients. Also, the incidence of PEP was 2-fold higher in the first 400 patients versus in the last 410 patients. The first 400 and next 410 cases age were $(61.15 \pm 18.22)$ and $(61.19 \pm 19.76)$ years, respectively, with no statistical difference ( $p=0.698$ ) (Table 3). This greater PEP incidence found among the first 400 patients of our series can be explained by the relative lack of experience and expertise of the endoscopist and the ERCP team. With increased experience, a reduced incidence of PEP was observed in the last 410 patients. Patients with mild or moderate PEP responded well to the medical therapy and their condition improved. One patient (0.12\%) with severe PEP died due to septic shock and multiorgan failure despite administration of current therapeutic methods. The scheme of prevention of pancreatitis after ERCP is shown in Figure 1. In our study, as risk factors include suspected sphincter of Oddi dysfunction (SOD), history of previous post-ERCP pancreatitis, peripapillary duodenal diverticu- 


\section{Table 2. Liver function test and WBC counts}

\begin{tabular}{|c|c|c|c|c|c|}
\hline \multirow[t]{2}{*}{ Variables } & \multicolumn{2}{|c|}{$\begin{array}{c}\text { Amylase }<300 \text { IU/L } \\
(n=525)\end{array}$} & \multicolumn{2}{|c|}{$\begin{array}{c}\text { Amylase }>300 \text { IU/L } \\
\quad(n=285)\end{array}$} & \multirow[t]{2}{*}{ p } \\
\hline & Mean & SD & Mean & SD & \\
\hline WBC & 11.33 & 6.23 & 14.03 & 8.67 & 0.001 * \\
\hline GGT & 340.1 & 318.71 & 414.62 & 353.25 & 0.001 * \\
\hline ALP & 200.79 & 141.8 & 221.86 & 221.79 & 0.774 \\
\hline Direct bilirubin & 3.12 & 3.4 & 3.94 & 4.09 & 0.001 * \\
\hline ALT & 187.31 & 188.96 & 251.28 & 313.77 & 0.001 * \\
\hline AST & 168.65 & 197.24 & 232.44 & 285.07 & 0.001 * \\
\hline
\end{tabular}

Table 3. Amylaseelevationand PEP in thefirst 400 andlast 410 patients

\begin{tabular}{|c|c|c|c|c|c|}
\hline \multirow[t]{2}{*}{ Variables } & \multicolumn{2}{|c|}{$\begin{array}{l}\text { First } 400 \text { patients } \\
\qquad(n=400)\end{array}$} & \multicolumn{2}{|c|}{$\begin{array}{l}\text { Last } 400 \text { patients } \\
\qquad(n=410)\end{array}$} & \multirow[t]{2}{*}{ p } \\
\hline & Mean & SD & Mean & SD & \\
\hline Age & 61.15 & 18.22 & 61.19 & 19.76 & 0.698 \\
\hline Amylase (IU/L) & 532.13 & 655.8 & 428.49 & 702.45 & 0.001 * \\
\hline Gender M/F, n & \multicolumn{2}{|c|}{$132 / 268$} & \multicolumn{2}{|c|}{$238 / 172$} & 0.001 * \\
\hline PEP Mild, $\mathrm{n}$ & \multicolumn{2}{|c|}{18} & \multicolumn{2}{|c|}{10} & 0.125 \\
\hline PEP Moderate, $\mathrm{n}$ & \multicolumn{2}{|c|}{12} & \multicolumn{2}{|c|}{5} & 0.089 \\
\hline PEP Severe, $\mathrm{n}$ & \multicolumn{2}{|c|}{1} & \multicolumn{2}{|c|}{0} & 0.494 \\
\hline
\end{tabular}

SD: Standard deviation; PEP. Post-ERCP pancreatitis; *Significant at 0.05 level; Mann-Whitney U test for numerical data, Fisher exact test for categorical data. Endoscopic retrograde cholangiopancreatography (ERCP); Post-ERCP pancreatitis (PEP); M: Male; F: Female.

lum and normal serum bilirubin. However, in our opinion as important factors affecting the risk of PEP include endoscopist experience, ERCP team, multidisciplinary approach, adequate deep sedation, a high-quality $\mathrm{C}$-arm $\mathrm{x}$ ray device and an experienced technician. We believe that several factors should be taken into account to prevent or minimize the development of PEP including endoscopist experience, ERCP team and ERCP room equipment.

Nineteen of the patients in our study were bleeding from papilla. Bleeding was controlled by sclerotherapy in 18 patients during ERCP. One patient had bleeding due to endoscopic sphincterotomy after ERCP. The bleeding was stopped endoscopically. Plastic stents were placed in 164 patients during ERCP procedure. There were no serious negative events associated with the stent. However, in 6 patients, proximal stent migration was detected, and it was removed using an endoscopic balloon. None of the patients had ERCP-induced cholangitis. Four patients were diagnosed with type 2 perforation (perforations around Ampulla Vateri) and responded positively to follow-up with medical treatment.

\section{Discussion}

The reported incidence of acute pancreatitis ranges from 0.4 to $1.5 \%$ with the use of diagnostic ERCP and from 1.6 to $5.4 \%$ with therapeutic ERCP. The incidence of severe acute pancreatitis associated with diagnostic ERCP varies between 0.4 and $0.7 \%$ and this risk may increase up to $20-40 \%$ in high-risk patients. ${ }^{[15]}$ Our study findings were consistent with previous literature data on PEP except for the incidence of severe acute pancreatitis of $0.12 \%$, which was lower than that reported in the literature. Additional risk factors reported for acute pancreatitis include the absence of bile duct dilation, bile duct diameter of $<1 \mathrm{~cm}$, 
Risk factors for Post-ERCP pancreatitis

Patient related factors (female gender, normal serum bilirubin, recurrent abdominal pain, and prior postERCP pancreatitis, recurrent pancreatitis or no chronic pancreatitis)

Endoscopist and Anesthesiologist experience (for deep sedation or general anesthesia)

Difficult cannulation, Transpancreatic septotomy, Precut sphincterotomy, Baloon dilation without prior biliary sphincterotomy

ERCP room equipment, High-quality $\mathrm{C}$ ann $\mathrm{X}$-ray imaging system and experienced $\mathrm{X}$-ray technician

Discussion of patient management with colleagues through internet applications (Friendly Team of Professional Doctors)

ERCP team, multidisiplinary approch

Figure 1. Prevention of pancreatitis after Endoscopic Retrograde Cholangio-Pancreatography.

younger age, difficult cannulation and unintentional pancreatography. ${ }^{[16]}$ Selective cannulation of the CBD is required for any therapeutic biliary intervention during endoscopic retrograde cholangiopancreatography (ERCP). Recently, an incremental increase was shown in the incidence of PEP with increasing cannulation attempts: $11.5 \%$ with $10-14$ attempts and $15 \%$ with $>15$ attempts. ${ }^{[17]} \mathrm{A}$ successful pre-cut sphincterotomy is not associated with an increased risk of adverse events. ${ }^{[18]}$ In our study, we observed an increased incidence of PEP with increasing cannulation attempts in the first 400 patients and among patients undergoing concomitant pre-cut sphincterotomy. Some studies compared amylase levels measured at 2, 3, 4, 6, 8 and 24 hours post-ERCP in order to define ideal timing for post-ERCP blood sampling and recommended 4-hour and 6-hour assessments from a practical point of view. ${ }^{[19,20]}$ Pancreatitis may develop even in painless patients with elevated post-ERCP amylase level. Uchino $\mathrm{R}$ et al. ${ }^{[21]}$ reported that computed tomography is useful to detect pancreatitis in patients taking analgesics, steroids or anti-immunological drugs and patients with diabetes mellitus and 18-hour serum amylase levels of $>6$ times greater than the upper limit of normal.

In our study, 24-h post-ERCP serum amylase levels were evaluated. Since ERCP was mostly performed at an outpatient setting without hospitalization of the patients in previous studies, 3-h and 4-h post-ERCP amylase levels were obtained from a practical standpoint. All of our patients were hospitalized for ERCP procedure. Thus, blood samples were collected within 24 hours of ERCP, i.e., at 06:00 hours next morning for measurement of amylase levels. Early use of pre-cut sphincterotomy was recommended to reduce adverse events associated with prolonged attempts at CBD cannulation. In experienced hands, an approach using primary precut appears to be at least as successful and safe as a conventional approach CBD cannulation. ${ }^{[22]}$ Pre-cut fistulotomy should represent rescue therapy after failure of standard cannulation. ${ }^{[23]}$ In a recent study evaluating patients undergoing ERCP under conscious sedation, it was found that one-third of patients experienced pain and discomfort during the procedure. Their data constitute powerful arguments for the use of deep sedation or general anesthesia in patients undergoing ERCP. ${ }^{[24]}$ An experienced anesthesia technician and an anesthesiologist are part of our ERCP team and we believe their key role in providing improved patient safety and satisfaction in the setting of ERCP contributes to reduction of PEP risk factors. Prospective study is always advantageous. Retrospective and single-centered study may be the lack of our article.

\section{Conclusion}

In conclusion, the risk of post-ERCP pancreatitis is multifactorial and the impact of some of the risk factors may be minimized or completely eliminated. Factors affecting the risk of PEP include endoscopist experience, ERCP team, multidisciplinary approach, adequate deep sedation, a high-quality $\mathrm{C}$-arm x-ray device and an experienced technician. It is our belief that endoscopist and his/her experience has a major role in avoiding or minimizing negative effects of these factors.

\section{Disclosures}

Ethichs Committee Approval: Our study is a retrospective study.

Peer-review: Externally peer-reviewed.

Conflict of Interest: None declared.

\section{References}

1. Jeurnink SM, Siersema PD, Steyerberg EW, Dees J, Poley JW, Haringsma J, et al. Predictors of complications after endoscopic retrograde cholangiopancreatography: a prognostic 
model for early discharge. Surg Endosc 2011;25:2892-900.

2. Lee YK, Yang MJ, Kim SS, Noh CK, Cho HJ, Lim SG, et al. Prediction of Post-Endoscopic Retrograde Cholangiopancreatography Pancreatitis Using 4-Hour Post-Endoscopic Retrograde Cholangiopancreatography Serum Amylase and Lipase Levels. J Korean Med Sci 2017;32:1814-9. [CrossRef]

3. Matsubara H, Urano F, Kinoshita Y, Okamura S, Kawashima $\mathrm{H}$, Goto $\mathrm{H}$, et al. Analysis of the risk factors for severity in post endoscopic retrograde cholangiopancreatography pancreatitis: The indication of prophylactic treatments. World J Gastrointest Endosc 2017;9:189-95. [CrossRef]

4. Cheung J, Tsoi KK, Quan WL, Lau JY, Sung JJ. Guidewire versus conventional contrast cannulation of the common bile duct for the prevention of post-ERCP pancreatitis: a systematic review and meta-analysis. Gastrointest Endosc 2009;70:1211-9. [CrossRef]

5. Cennamo V, Fuccio L, Zagari RM, Eusebi LH, Ceroni L, Laterza $\mathrm{L}$, et al. Can a wire-guided cannulation technique increase bile duct cannulation rate and prevent post-ERCP pancreatitis?: A meta-analysis of randomized controlled trials. Am J Gastroenterol 2009;104:2343-50. [CrossRef]

6. Lerch MM. Classifying an unpredictable disease: the revised Atlanta classification of acute pancreatitis. Gut 2013;62:2-3.

7. Kim J. Training in Endoscopy: Endoscopic Retrograde Cholangiopancreatography. Clin Endosc 2017;50:334-9.

8. Rabenstein T, Hahn EG. Post-ERCP pancreatitis: is the endoscopist's experience the major risk factor?. JOP 2002;3:17787.

9. Hellier MD, Morris AI. ERCP training--time for change. Gut 2000;47:459-60. [CrossRef]

10. Thaker AM, Mosko JD, Berzin TM. Post-endoscopic retrograde cholangiopancreatography pancreatitis. Gastroenterol Rep (Oxf) 2015;3:32-40. [CrossRef]

11. Liao Z, Hu LH, Li ZS, Zuo CJ, Wang L, Jin G, et al. Multidisciplinary team meeting before therapeutic ERCP. A prospective study with 1,909 cases. J Interv Gastroenterol 2011;1:64-9.

12. Williams EJ, Taylor S, Fairclough P, Hamlyn A, Logan RF, Martin D, et al. Are we meeting the standards set for endoscopy? Results of a large-scale prospective survey of endoscopic retrograde cholangio-pancreatograph practice. Gut 2007;56:821-9. [CrossRef]

13. Davee T, Garcia JA, Baron TH. Precut sphincterotomy for se- lective biliary duct cannulation during endoscopic retrograde cholangiopancreatography. Ann Gastroenterol 2012;25:291302.

14. Lopes L, Dinis-Ribeiro M, Rolanda C. Early precut fistulotomy for biliary access: time to change the paradigm of "the later, the better"?. Gastrointest Endosc 2014;80:634-41. [CrossRef]

15. El Nakeeb A, El Hanafy E, Salah T, Atef E, Hamed H, Sultan $\mathrm{AM}$, et al. Post-endoscopic retrograde cholangiopancreatography pancreatitis: Risk factors and predictors of severity. World J Gastrointest Endosc 2016;8:709-15. [CrossRef]

16. Arata S, Takada T, Hirata K, Yoshida M, Mayumi T, Hirota M, et al. Post-ERCP pancreatitis. J Hepatobiliary Pancreat Sci 2010;17:70-8. [CrossRef]

17. Choudhary A, Winn J, Siddique S, Arif M, Arif Z, Hammoud $\mathrm{GM}$, et al. Effect of precut sphincterotomy on post-endoscopic retrograde cholangiopancreatography pancreatitis: a systematic review and meta-analysis. World J Gastroenterol 2014;20:4093-101. [CrossRef]

18. Navaneethan U, Konjeti R, Lourdusamy V, Lourdusamy D, Mehta D, Sanaka MR, et al. Precut sphincterotomy: efficacy for ductal access and the risk of adverse events. Gastrointest Endosc 2015;81:924-31. [CrossRef]

19. Testoni PA, Caporuscio S, Bagnolo F, Lella F. Twenty-four-hour serum amylase predicting pancreatic reaction after endoscopic sphincterotomy. Endoscopy 1999;31:131-6. [CrossRef]

20. Ito K, Fujita N, Noda Y, Kobayashi G, Horaguchi J, Takasawa 0 , et al. Relationship between post-ERCP pancreatitis and the change of serum amylase level after the procedure. World J Gastroenterol 2007;13:3855-60. [CrossRef]

21. Uchino R, Sasahira N, Isayama H, Tsujino T, Hirano K, Yagioka $\mathrm{H}$, et al. Detection of painless pancreatitis by computed tomography in patients with post-endoscopic retrograde cholangiopancreatography hyperamylasemia. Pancreatology 2014;14:17-20. [CrossRef]

22. de Weerth A, Seitz U, Zhong Y, Groth S, Omar S, Papageorgiou $C$, et al. Primary precutting versus conventional overthe-wire sphincterotomy for bile duct access: a prospective randomized study. Endoscopy 2006;38:1235-40. [CrossRef]

23. Ayoubi M, Sansoè G, Leone N, Castellino F. Comparison between needle-knife fistulotomy and standard cannulation in ERCP. World J Gastrointest Endosc 2012;4:398-404. [CrossRef]

24. Thosani N, Banerjee S. Deep sedation or general anesthesia for ERCP?. Dig Dis Sci 2013;58:3061-3. [CrossRef] 